UROLOGICAL CANCER

\section{Atezolizumab: an alternative to cisplatin?}

Cisplatin-based chemotherapy is an effective first-line treatment of patients with locally advanced or metastatic urothelial carcinoma; however, many patients are ineligible to receive cisplatin, and other types of chemotherapy, although better tolerated, are not as effective. Now, data from a single-arm phase II trial indicate that treatment with the anti-programmed cell death 1 ligand 1 (PD-L1) antibody atezolizumab provides an alternative approach for patients with locally advanced or metastatic urothelial carcinoma who are not eligible to receive cisplatin.

Describing the key features of this trial, lead author Arjun Balar explains how changes in the trial design over time led to the final approach: "The original design included cohort one as an exploratory cohort of 30 cisplatinineligible patients, which was intended to get a sense of the activity and safety of atezolizumab in the first-line setting. When emerging data from the second-line group of the same study (cohort two) were promising, cohort one was expanded in size to approximately 100 patients". A total of 119 patients received 3-weekly intravenous doses of atezolizumab until evidence of disease progression emerged.

Treatment with atezolizumab resulted in a median overall survival duration of 15.9 months, with an objective response rate of $23 \%$, rising to $28 \%$ in patients with the highest levels of PD-L1 expression on tumour-infiltrating immune cells: "the response rate and median survival in this group, albeit in a non-randomized trial, were extremely promising" highlights Balar. The majority (19 of 27 patients) who responded to

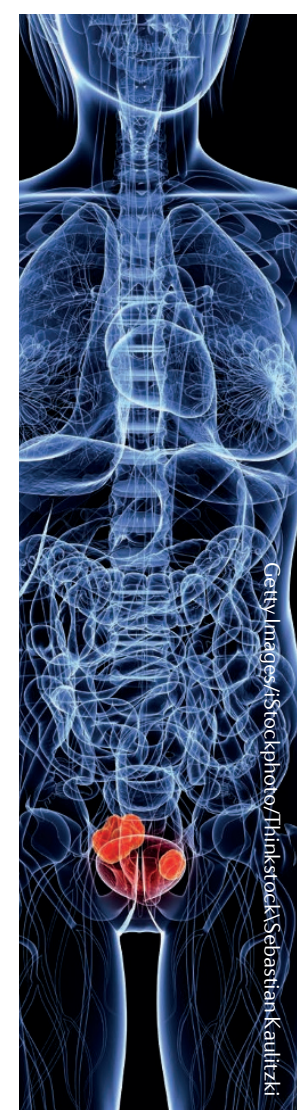

treatment had an ongoing response at the time of analysis. Furthermore, these patients were found to have a significantly higher mean mutation load than those who did not respond to treatment.

Adverse events of any grade occurred in $66 \%$ of patients; however, only $16 \%$ had grade 3-4 adverse events, a rate much lower than is typically associated with treatment with carboplatin, the most commonly used alternative to cisplatin in these patients.

"The implications of our current study, when put in the context of other trials of immunotherapy in urothelial cancer, are that immune checkpoint inhibitors will be firmly established in the treatment armamentarium for this disease" concludes Balar, who adds that phase III trials, exploring this approach in a comparative way, are ongoing.

Peter Sidaway

ORIGINAL ARTICLE Balar, A. V. et al.

Atezolizumab as first-line treatment in cisplatinineligible patients with locally advanced and metastatic urothelial carcinoma: a single-arm, multicentre, phase 2 trial. Lancet http://dx.doi.org/ 10.1016/S0140-6736(16)32455-2 (2016) 